Boise State University

ScholarWorks

$12-1-2012$

\title{
Force-Feedback High-Speed Atomic Force Microscope for Studying Large Biological Systems
}

Byung I. Kim

Boise State University

Ryan Boehm

Boise State University 


\title{
Force-Feedback High-Speed Atomic Force Microscope for Studying Large Biological
}

\author{
Systems \\ Byung I. Kim* and Ryan D. Boehm \\ Department of Physics, Boise State University \\ 1910 University Drive, Boise, Idaho
}

We designed and developed a high-speed atomic force microscope (HSAFM) utilizing a forcefeedback scheme for imaging large biological samples. The system collects three simultaneous images: a deflection image, a topographic image, and a force image. We demonstrated that this force-feedback HSAFM is capable of acquiring large topographic images of Escherichia coli biofilms at approximately one frame per second in air. We discuss how the self-actuating cantilever and the piezo tube follow those larger biological topographic features during the HSAFM imaging process.

*Corresponding Author: ByungKim@boisestate.edu

PACS Number: 68.35.-p; 68.37.-d; 68.37.Ps; 68.47.-b

Key Words: COIFM; force-feedback; high-speed AFM; interfacial force microscope; biological AFM; biological dynamics

Non-Common Abbreviations: high-speed atomic force microscopy (HSAFM); cantilever-based optical interfacial microscope (COIFM); dimensional micro-actuated silicon probe (DMASP) 


\section{Introduction}

The recent advancement of high-speed atomic force microscopy (HSAFM) has enabled researchers to view the nanometer-scale dynamic behavior of individual biological and biorelevant molecules at a molecular-level resolution under physiologically relevant time scales (Ando et al., 2001; Crampton et al., 2007; van Noort et al., 1999), which is the realization of a dream in the life sciences. Such studies include the visualization of various dynamic activities carried out by biological macromolecules (Ando et al., 2008a; Ando et al., 2008b), such as motor proteins and cytoskeletal fibers (Ando et al., 2001), nucleic acids/proteins in real time (Crampton et al., 2007), and biopolymers (van Noort et al., 1999; Viani et al., 1999). The enhancement of the scan speed, development of a high z-bandwidth feedback loop, and the advancement of tapping-mode imaging with a small cantilever make it possible to improve the resolution of topographic signals in both time and space in less invasive ways (Ando et al., 2001; Ando et al., 2008a; Ando et al., 2008b; Crampton et al., 2007; Humphris et al., 2005; Kodera et al., 2006; Picco et al., 2007; van Noort et al., 1999, Zou et al., 2008).

These high-speed imaging applications now extend to the cellular/bacterial systems with the use of a smaller cantilever (Fantner et al., 2010; Hansma et al. 2006). Fantner et al. viewed that the limitation originates from the size of the cantilever, as the cantilever response speed is proportional to the cantilever resonance frequencies (Viani et al., 1999; Fantner et al., 2010). The use of a cantilever with high-resonant vibrational frequency allows more rapid vertical tip movement, thus obtaining high-speed imaging. By reducing the size of the cantilevers of HSAFM to a width of $10 \mu \mathrm{m}$ and a length of 20-30 $\mu \mathrm{m}$ (Fantner et al., 2010), instead of using conventional cantilevers that are tens of $\mu \mathrm{m}$ wide and hundreds of $\mu \mathrm{m}$ long, the system has demonstrated image speeds up to one frame per second for small biomolecular structures such as 
DNA and one frame per ten seconds for larger biological systems, such as bacteria (Fantner et al., 2010; Hansma et al. 2006; Viani et al., 1999). To understand many rapid large-scale biological phenomena, this imaging speed is not sufficient enough. For instance, the roughening variation of bacteria occurs within a few seconds in response to an antimicrobial (Fantner et al., 2010; Katan and Dekker, 2011). Also, the use of smaller cantilevers creates some challenges, such as fabrication and signal detection with a smaller laser spot size (Ando et al., 2001; Walters et al., 1996).

Here we introduce an alternative approach to that of employing a small cantilever for high-speed imaging, called "force-feedback" HSAFM. This new system utilizes our recent development of a cantilever-based optical interfacial microscope (COIFM) (Bonander and Kim, 2008; Kim et al., 2011a; Kim et al., 2011b). Rather than using a smaller cantilever, we still use a conventional-size self-actuation cantilever that is capable of fast response through the force-feedback mechanism. The force-feedback shortens the response time of the sensor, which is the most essential component for this HSAFM. Not only does it allow for the high-speed imaging, it has a displacement capability of micrometers through a piezo tube, thus enabling the system to extend to applications ranging from biomolecules to cellular systems. This novel force-feedback HSAFM will contribute greatly to the studies of these large-scale biological phenomena (Fantner et al., 2010; Hansma et al. 2006; Katan and Dekker, 2011) through the improvement of the time resolution for scan sizes up to several micrometers.

\section{Design Concept and Set-Up}

The conventional AFM has used a flexible cantilever to have higher sensitivity and to make the probe less invasive to soft sample surfaces. However, this approach has a drawback in highspeed imaging on these surfaces: Assuming that the biological sample can be considered as a soft 
medium with viscoelastic properties, a typical transient response of the cantilever with time $(t)$

for a disturbance force during the force-feedback can be described by a simple exponential decay function or $1-\exp \left(-\omega_{0} t / Q\right)$, where $\omega_{0}$ is an angular frequency at resonance and $Q$ is quality factor (Albrecht et. al., 1991; Sulchek et al., 2000). Since the imaging speed is determined by the decay constant $\tau=Q / \omega_{0}$, the lower resonance angular frequency $\omega_{0}$ of the soft cantilever causes the slow imaging speed. To improve the imaging speed, either the resonance frequency or the spring constant should be large. Therefore, the COIFM is an ideal tool to make the spring constant large, because the spring constant can be considered infinite due to the counter force. This is because the COIFM has zero compliance, or $\Delta \mathrm{z}$ equals zero, and the spring constant is defined as $k=\Delta F / \Delta z$, according to Hooke's law (i.e. $F=k \cdot z$ ). By employing a feedback scheme to induce a counter force, the cantilever is able to retain a normal size, and therefore avoid the fabrication and signal detection issues that are related to smaller cantilever sizes. This concept leads to our novel force-feedback HSAFM system for fast biological imaging.

We utilized our existing COIFM system capability (originally designed for interfacial force measurements) as a base to develop our force-feedback system. The force-feedback HSAFM requires 1) fast response of the force-feedback loop; and 2) capability of tracking large topographic features on biological surfaces. This fast response was realized due to the highresonance frequency of the cantilever with a built-in actuator (a bimorph stack). Tracking larger topographic variations was accomplished by employing a conventional piezo tube. Figure 1 shows the conceptual design of the force-feedback HSAFM and how it operates during the imaging process step-by-step. When the tip encounters a larger topographic feature of a biological sample during scanning (see step 1), the force exerted upon the tip causes it to bend upwards (see step 2). An error signal $\left(\mathrm{V}_{\text {Error }}\right)$ generated by the bending motion of the cantilever is 
removed by a counter force applied to the self-actuating cantilever through force-feedback. The soft biological sample is compressed and deformed during this process (as shown in step 3). Additionally, the signal is sent to the piezo tube through another feedback loop. It is important to note that both the actuations of the cantilever and the piezo tube can occur simultaneously and cooperatively. However, the response time of the piezo tube is roughly ten times slower than the cantilever and therefore the high speed imaging rate is mainly limited by the response time of the feedback loop that involves the piezo tube. The response time corresponds to the transition time from step 3 to step 4, where the piezo tube has moved down to release compression and deformation of soft biological samples.

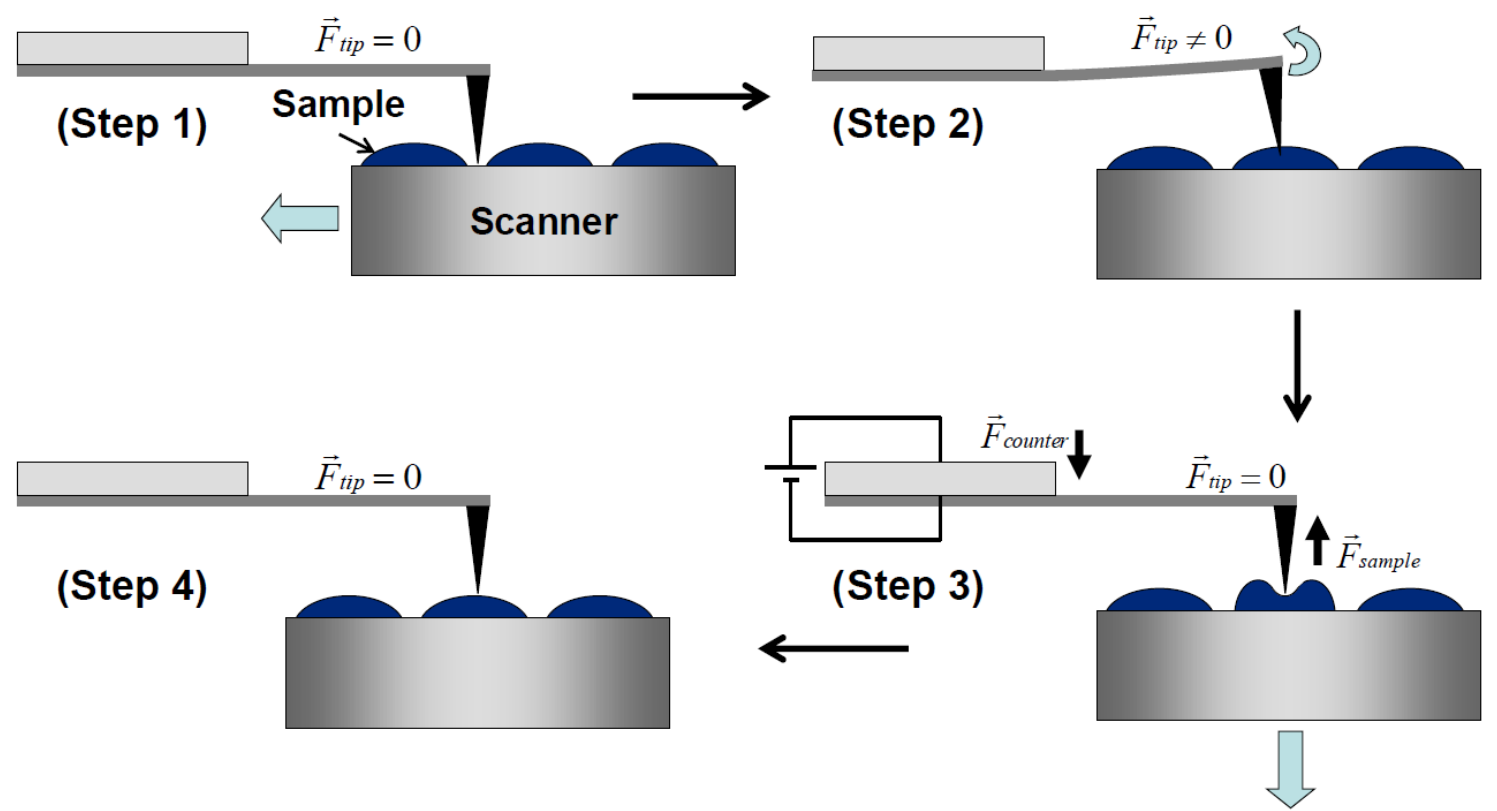

Figure 1. Conceptual diagrams for the principle behind the force-feedback HSAFM. The tip is in contact with the sample surface without the bending of the cantilever (Step 1). The tip encounters the biological structure when the sample moves laterally, causing the tip-bending (Step 2). When a voltage is applied to the $\mathrm{ZnO}$ stack due to the force-feedback, the counter force compresses and deforms the biological structure (Step 3). The biological structure is released from compression and deformation, as the sample moves down vertically due to the activation of feedback loop involving the piezo tube (Step 4). 


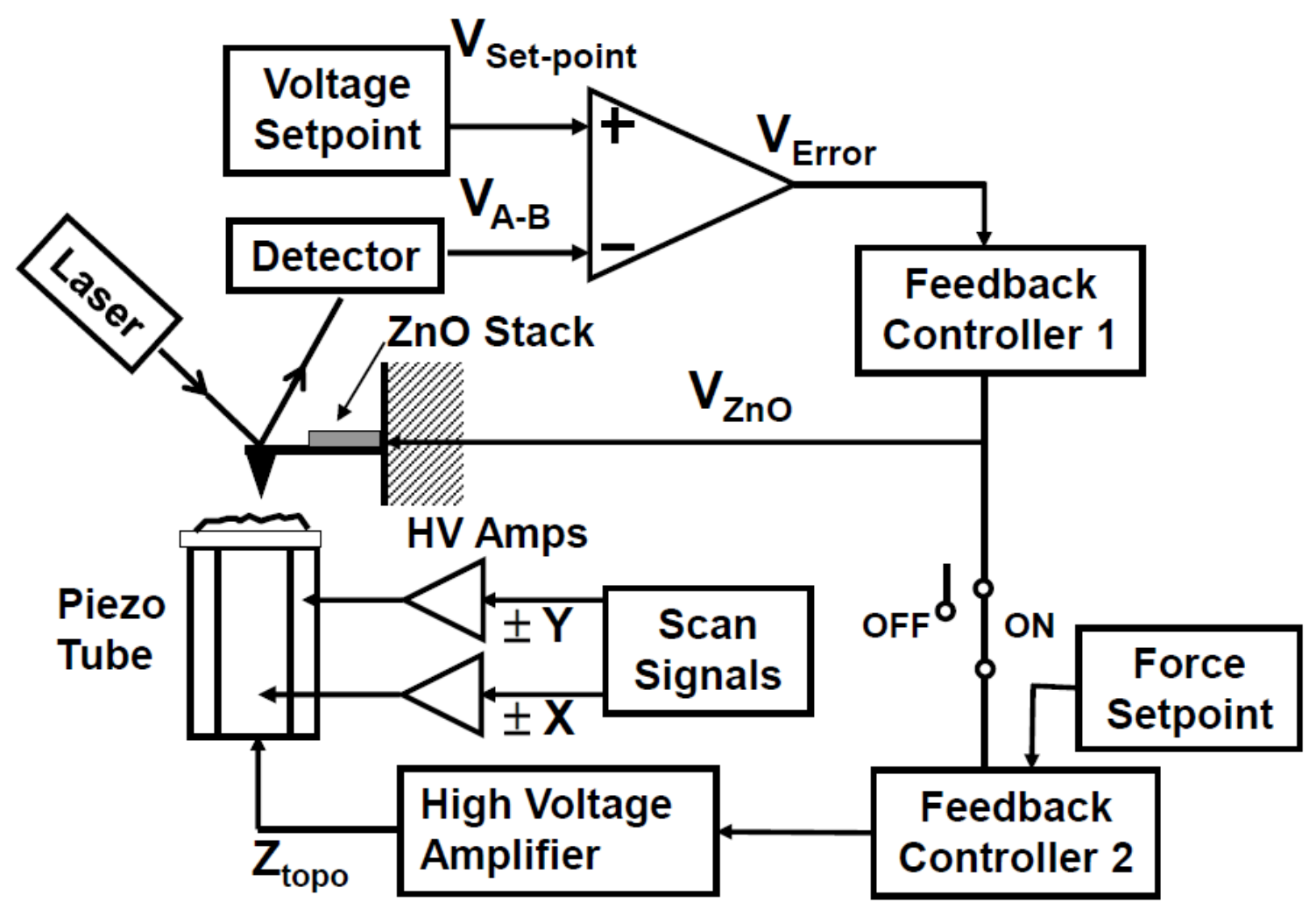

Figure 2. A schematic diagram of the force-feedback HSAFM system. The system employs an optical beam deflection detection scheme, force-feedback loop, topographic feedback loop, and a piezo tube for XY scanning and Z-feedback. The force-feedback HSAFM generates three different images: a deflection image $\left(\mathrm{V}_{\mathrm{A}-\mathrm{B}}\right)$, a topographic image $\left(\mathrm{Z}_{\mathrm{Topo}}\right)$, and a force image $\left(\mathrm{V}_{\mathrm{ZnO}}\right)$. The feedback controller 1 is for the force-feedback loop for force images, whereas the feedback controller 2 is for the topographic feedback loop involving the piezo tube. The ON/OFF switch is for enabling and disabling of the topographic feedback loop.

We developed the force-feedback HSAFM using a commercially available cantilever called a "dimensional micro-actuated silicon probe" (DMASP) for both force-sensing and actuation for the force-feedback. The DMASP cantilever has nominal dimensions of $55 \mu \mathrm{m}$ width and $125 \mu \mathrm{m}$ length (Veeco, 2006). These dimensions are several times larger than the small-sized cantilever dimensions employed in existing HSAFMs. By using this larger dimensional cantilever, we can use standard AFM cantilever holders and laser beams for HSAFM development using ordinary AFM systems. This compatibility with the ordinary AFM will make the force-feedback HSAFM 
easy to adapt, avoiding the fabrication and signal detection issues associated with the use of small cantilevers. The DMASP probe contains a $\mathrm{ZnO}$ stack with a thickness of $3.5 \mu \mathrm{m} \mathrm{ZnO}$ sandwiched between two $0.25 \mu \mathrm{m}$ Ti/Au layers, providing its nominal force constant of $\sim 3 \mathrm{~N} / \mathrm{m}$ (Veeco, 2008). This stack with the silicon cantilever has been used for fast imaging due to its higher-resonant frequency (nominally around $50 \mathrm{kHz}($ Veeco, 2008)) over the piezo tube actuator ( 10 kHz) in ordinary AFMs (Manalis et al., 1996a, Manalis et al., 1996b; Minne et al., 1995; Rogers et al., 2002; Rogers et al., 2003; Sulchek et al., 1999). The force-feedback loop consisted of the DMASP, a feedback controller, and an AutoProbe LS head (Park Scientific Instruments) as shown in Figure 2. The DMASP sensor is wire-bonded to its built-in holder as received, and is placed in the AFM cartridge for the laser beam alignment. A modified Burleigh STM controller (Burleigh Instruments Inc., Fishers, NY) was used as the force-feedback controller. The built-in optical beam deflection detection scheme was used to transmit the interaction force between the tip and the surface into the electrical signal (Meyer et al., 1988). In the laser beam deflection detection system, a position-sensitive photo-detector (PSPD) was adjusted to align the laser beam (deflected from the cantilever) to be positioned at its center. The PSPD output, $\mathrm{V}_{\mathrm{A}-\mathrm{B}}$ signal, was connected to the feedback input of the controller. The feedback control parameters, such as time constant and gain, were manually adjusted for an optimal feedback condition. The output of the feedback controller was sent directly to the $\mathrm{ZnO}$ stack of the DMASP to apply a counter force to the cantilever. The actuation function of the DMASP was used to control the force applied to the cantilever to maintain the constant deflection of the cantilever at a set-point value during the data acquisition. The applied force was obtained by multiplying the output voltage of the feedback controller with the conversion factor, $5 \mathrm{nN} / \mathrm{V}$, reported in our earlier publication (Kim et al., 2011a). Because the breakdown voltage of the 
ZnO actuator DMASP is nominally $\pm 10 \mathrm{~V}$ (Veeco, 2008), the controller output was designed to be saturated for the force variations larger than $50 \mathrm{nN}$ during the data acquisition.

An additional feedback loop involving the piezo tube was developed for large-scale topographic imaging capabilities. In the feedback loop, the output of the force-feedback controller was fed through an RHK SPM 100 controller (RHK Technology Inc. Troy, MI) via an ON/OFF switch (see Figure 2). The output of the RHK controller was sent to the z-electrode of the piezo tube via high-voltage amplifier $(\times 22.5)$. The feedback loop maintains the force signal constant by changing the tip-sample spacing up to $\sim 1.5 \mu \mathrm{m}$ in the $z$ direction using the piezo tube, sufficient to follow typical biological cells. The voltage applied to the piezo tube was recorded as a topographic signal for each AFM image displayed. All data was recorded through analog-todigital converter (ADC) inputs of the RHK 100 and XPM PRO software. The X and Y scanners of the piezo tube were used for the sample movement, which were controlled by the high voltage scan signals. The maximum scanning area of the system is $100 \mu \mathrm{m} \times 100 \mu \mathrm{m}$.

\section{Experimental}

In this experiment, we set the force to $\sim 0 \mathrm{nN}$ with the activation of the feedback loop during HSAFM imaging. All data was collected on Escherichia coli (E. coli) culture and biofilm, which were taken from a PBS solution and deposited on a standard two-dimensional grating with 10 $\mu \mathrm{m}$ periodicity (laterally) and $180 \mathrm{~nm}$ step height acquired from Veeco Inc. (Veeco, 2008). The culture of a non-pathogenic strand of E. coli strain (RK4353) was grown overnight with shaking (225 rpm) at $37^{\circ} \mathrm{C}$ in $5 \mathrm{~mL}$ of Luria Bertani broth. The culture was pelleted at $6000 \mathrm{xg} / 10 \mathrm{~min}$, and resuspended in sterile PBS. Then $1 \mathrm{~mL}$ was dispensed into $99 \mathrm{~mL}$ of fresh PBS solution causing a 1:100 part dilution. The sample was surveyed using the optical imaging of a vertically 
placed, charge-coupled device (CCD) camera with two different objective magnifications $(20 \times$ and $80 \times$ ), before and after dropping $1 \mathrm{~mL}$ of the diluted $E$. coli and related biofilm solution on the grating sample. The lower magnification provides a large overview of the dispensed material, whereas the higher magnification provides a detailed view within the dispensed material. The 10 $\mu \mathrm{m}$ periodicity and $200 \mathrm{~nm}$ step height of the grating sample were used for an additional dimensional scale during imaging. Deflection, force, and topographic images were taken at $0 \mathrm{nN}$ set-force for various scan speeds and scan sizes on the biofilm and $E$. coli samples.

\section{Result and Discussion}
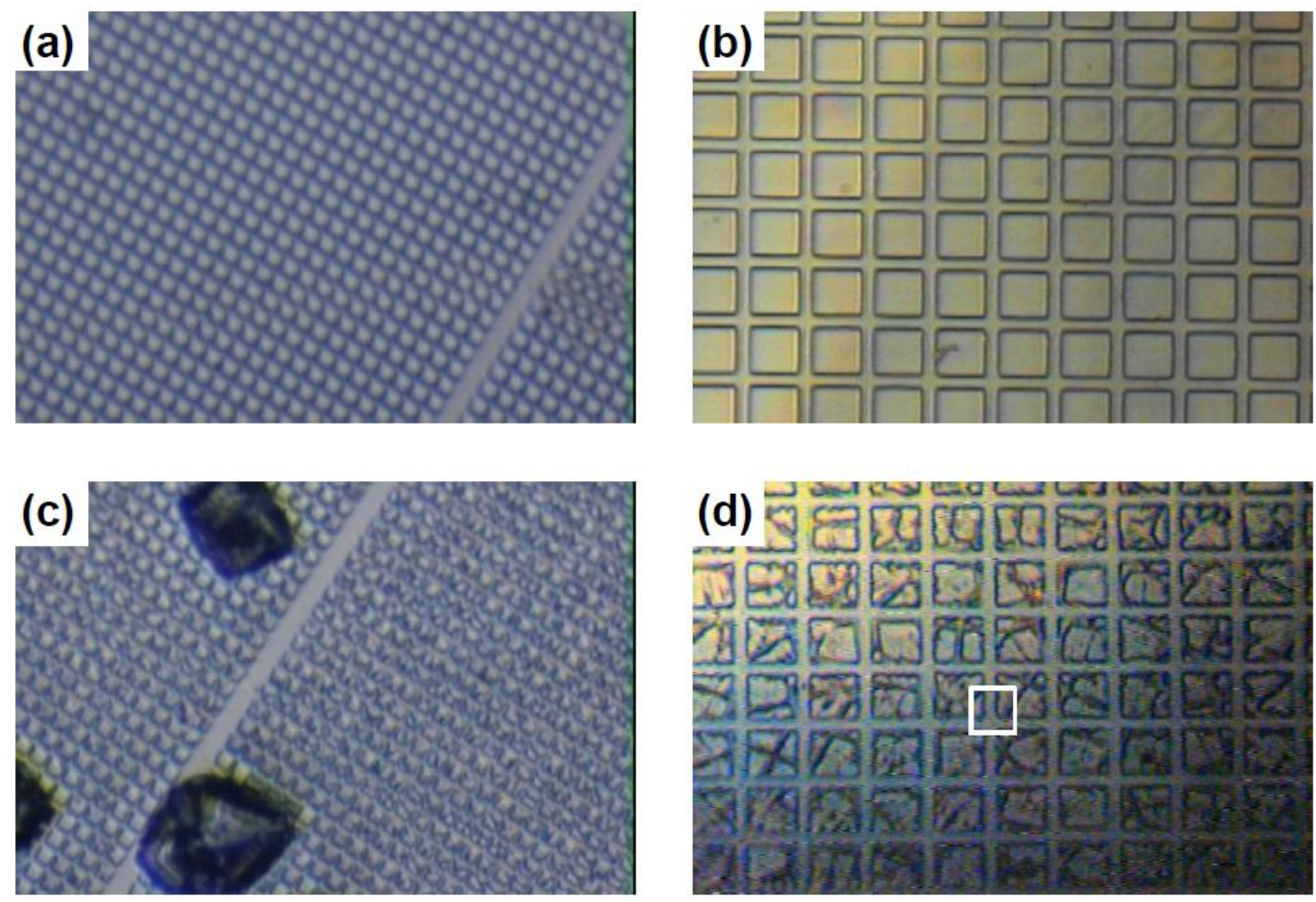

Figure 3. Magnified optical images of a clean two-dimensional grating with $10 \mu \mathrm{m}$ periodicity using a 20x objective lens (a) and an 80x objective lens (b). Magnified optical images of E. coli bacteria and their biofilms deposited on the grating using a 20x objective lens (c) and an 80x objective lens (d). E. coli biofilms in the PBS solution were collected, deposited, and dried on the grating surface. A square outlined with white lines is the area where the images were taken subsequently by the forcefeedback HSAFM. 
Figures 3(a) and 3(b) show lower and higher magnification optical images, respectively, before applying the E. coli biofilms on the clean grating sample surface. The square-like periodic patterns are clearly visible. These patterns provide not only the reference to determine if the $E$. coli film is deposited, but also give a scale for the images of interest. Figure 3(c) shows a low magnified optical image of the E. coli biofilms on the sample surface. Large pyramidal-shape structures were observed in the optical image, which are most likely artifacts due to the crystallization of ions in the PBS solution as the solution dried. Figure 3(d) shows a higher magnified optical image of the E. coli biofilms on the sample surface. By comparing the before and after images, it was evident that material had been trapped in the grating indents. Therefore, the optical images allow for the tip to be moved to a desirable location to be compared with the high-speed images to understand the general distribution of the films.
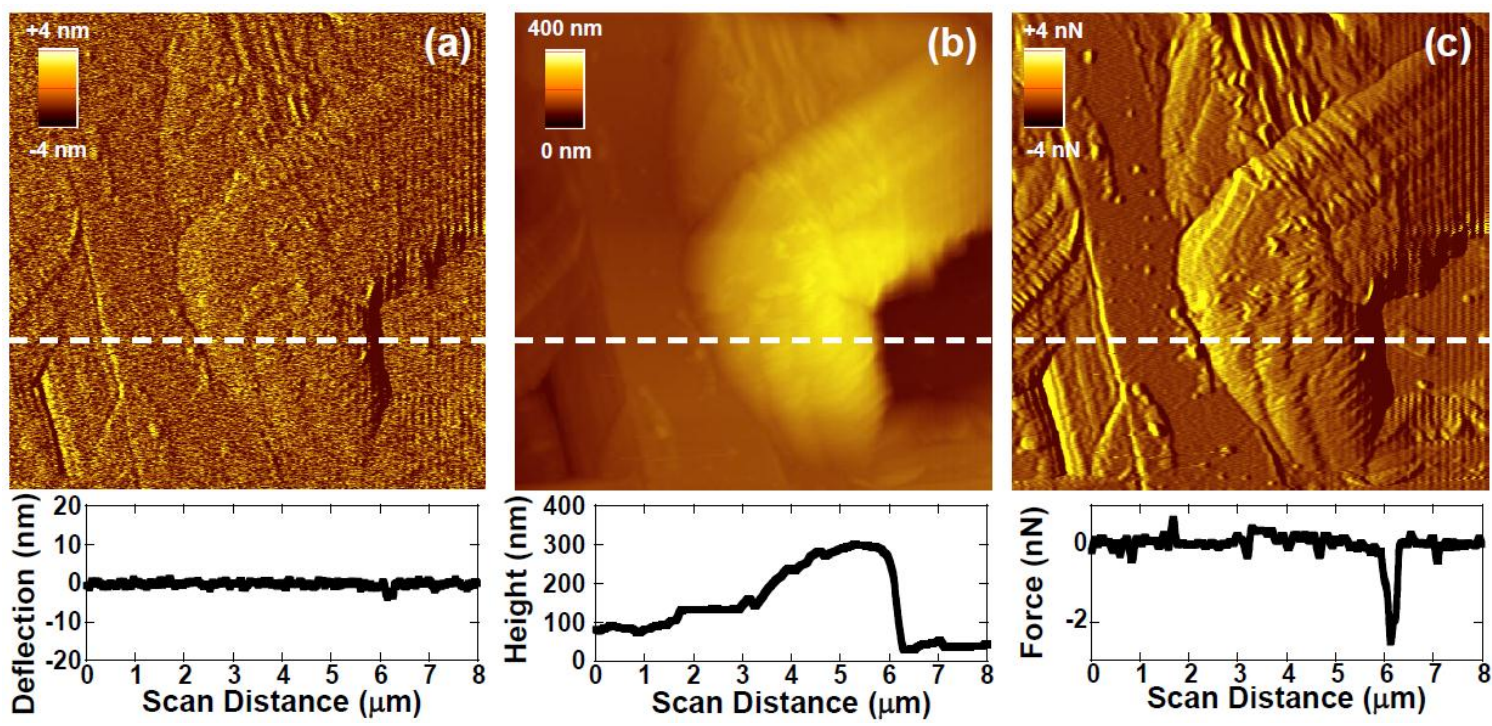

Figure 4. Three images taken by the force-feedback HSAFM on the E. coli biofilms on the grating. (a) Deflection image $\left(\mathrm{V}_{\mathrm{A}-\mathrm{B}}\right)$. (b) Topographic image $\left(\mathrm{Z}_{\mathrm{Topo}}\right)$. (c) Force image $\left(\mathrm{V}_{\mathrm{ZnO}}\right)$. Images were collected at $0 \mathrm{nN}$ set-force on the grating structure at a scan rate of $20 \mathrm{~ms} / \mathrm{line}$ (scan area: $8 \mu \mathrm{m} \times 8 \mu \mathrm{m}$ ). The lower panels represent sectional profiles taken along the dashed lines. 
The principle of force-feedback HSAFM was tested by imaging biological samples with large height variations on a two-dimensional grating with $10 \mu \mathrm{m}$ periodicity. The tip was scanned in a square outlined with white lines, as seen in Figure 3(d). Minimal signal changes less than $1 \mathrm{~nm}$ were exhibited in $\mathrm{V}_{\mathrm{A}-\mathrm{B}}$, as shown in Figure 4(a). This is due to the actuation voltages being applied to the piezo tube and the $\mathrm{ZnO}$ stack, as displayed in Figure 4(b) and Figure 4(c), respectively. This result suggests that both force-feedback and piezo tube work together cooperatively to make $\mathrm{V}_{\mathrm{A}-\mathrm{B}}$ approximately zero. Since the system originally records $\mathrm{V}_{\mathrm{A}-\mathrm{B}}$ and $\mathrm{V}_{\mathrm{ZnO}}$ in volts, the conversion factors $1 \mathrm{~nm} / 20.4 \mathrm{mV}$ and $5 \mathrm{nN} / \mathrm{V}$ were used, respectively, to turn the signals into deflection and force images (Kim et al., 2011a). The minimal signal change in the "deflection image" from $\mathrm{V}_{\mathrm{A}-\mathrm{B}}$ of Figure $4(\mathbf{a})$ and its underlying sectional profile suggest the feedback loop is efficiently working to compensate for the error and that the system is following the sample topographic variations accurately. Since the voltage applied to the piezo tube is related to the amount of the displacement of the sample, Figure 4(b) represents a "topographic image." The image was taken on the biological surface with a scan area of $8 \mu \mathrm{m} \times 8 \mu \mathrm{m}$ at the condition of $\mathrm{V}_{\mathrm{A}-\mathrm{B}}$ signal zero at a scan speed of $20 \mathrm{~ms} / \mathrm{line}$. Part of the periodic grating structure (see in Figure 3) can be observed on the topographic image. The sectional profile in Figure 4(b) shows that the topographic height variations ranged $300-400 \mathrm{~nm}$ during high-speed imaging. It appears that the biofilm and its corresponding extracellular polymeric substance have been trapped in the lower part of the grating sample, as seen by the large feature with a sudden increase of topographic height. Fine structure roughness is evident on the surface of the biofilm as seen by the elevated texturing of stripe-like features along the diagonal direction of the surface with a periodicity around $\sim 500-800 \mathrm{~nm}$ with a height of $\sim 50 \mathrm{~nm}$. The biofilms appear to be buckled due to the grating structure and in an " $\mathrm{L}$ "-shape. The flat area is the upper terrace of the 
grating sample where the diameter of some small structures (most likely debris of the biofilm from the transplantation process) ranges between $10 \mathrm{~nm}$ and $30 \mathrm{~nm}$. The biofilm is unevenly spread. The underlying panel shows a sectional profile of biofilm (with a topographic height of $\sim 180 \mathrm{~nm}$ ) and grating sample along the dashed line, showing that the force-feedback HSAFM follows subtle structural variations accurately. Figure 4(c) represents a "force image" from the $\mathrm{V}_{\mathrm{ZnO}}$, a force-feedback signal in conjunction with topographic feedback to compensate for the $\mathrm{V}_{\mathrm{A}-\mathrm{B}}$ signal shown in the deflection image, Figure 4(a). Interestingly, the image shows an enhanced contrast over the topographic images, especially near a boundary where the two different structures meet, allowing for clearer visualization of the small features just described in relation to Figure 4(b). This idea is supported by the fact that the underlying sectional profile of Figure 4(c) shows one large valley, indicating a large topographic change. When the force sectional profile is related to the topographic sectional profile, the large valley in the force signal is indicated to be due to the large topographic height of the biofilm decreasing abruptly to that of the lower terrace of the grating sample. The result suggests that the sudden signal changes near such abrupt structures are too fast for the topographic feedback involving the piezo tube to attain the set-force through the removal of the feedback error. The minimally observed change in the deflection image indicates that these uncompensated signals are delivered and compensated by the force-feedback involving the fast cantilever to make the deflection signal stay constant. Therefore, both feedbacks worked cooperatively in cancelling the deflection signal in response to the surface variations, as discussed in diagrams of Figure 1. The force-feedback loop allows the self-actuated cantilever to respond rapidly, while the topographic-feedback loop allows the piezo tube to relieve the compression and deformation created by the cantilever to attain a constant force on the cantilever through the force-feedback loop (see steps 3 and 4 in Figure 1). It seems 
that this cooperative feedback compensation enables the cantilever to follow the topographic features of biological samples with negligible bending.
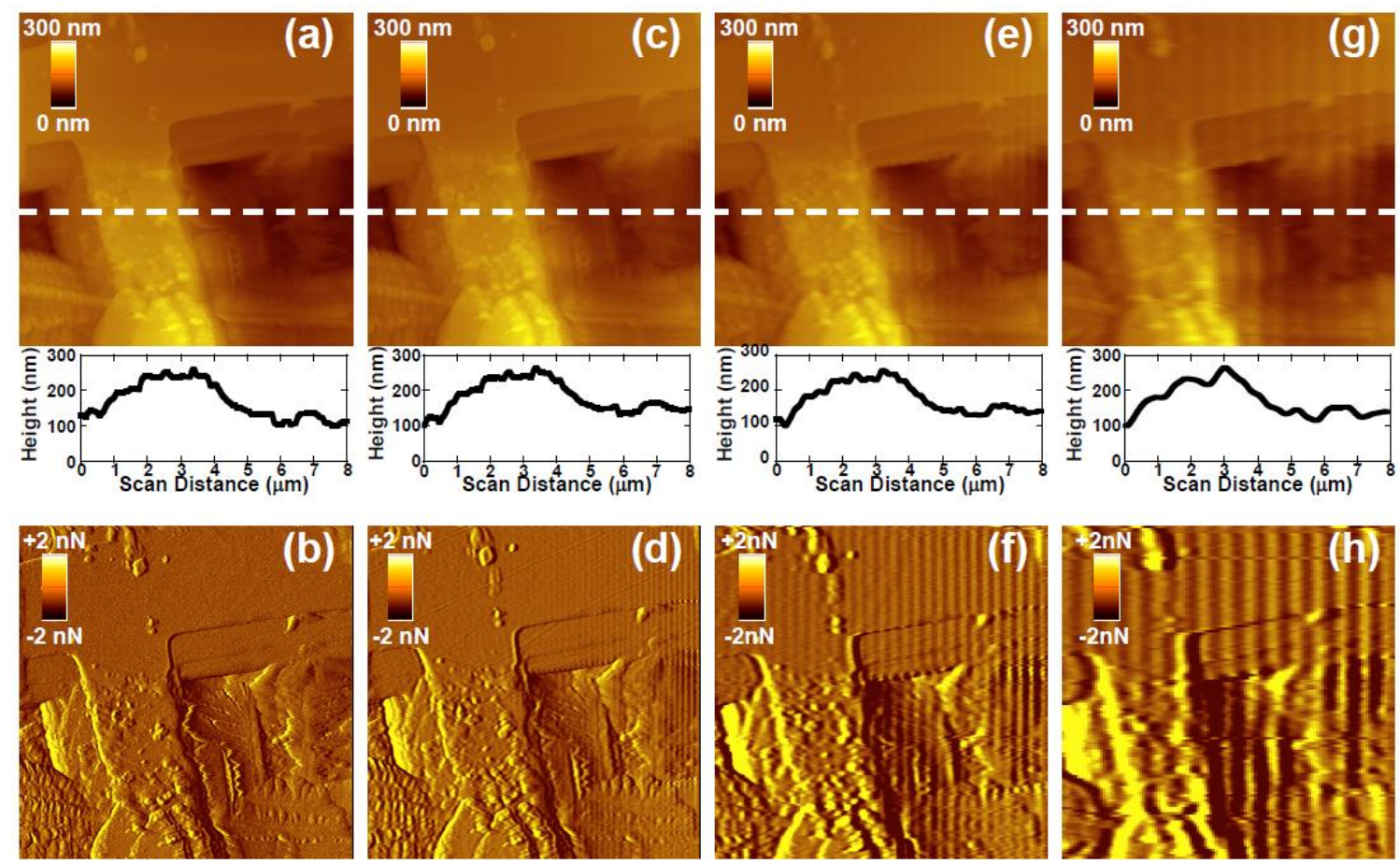

Figure 5. Topographical and force images collected simultaneously at $0 \mathrm{nN}$ set-force on the E. coli biofilms at four different scan rates: $50 \mathrm{~ms} / \mathrm{line}(\mathbf{a}, \mathbf{b}), 20 \mathrm{~ms} / \mathrm{line}(\mathbf{c}, \mathbf{d})$, $10 \mathrm{~ms} / \mathrm{line}(\mathbf{e}, \mathbf{f})$, and $5 \mathrm{~ms} /$ line $(\mathbf{g}, \mathbf{h})$. The scan area is $8 \mu \mathrm{m} \times 8 \mu \mathrm{m}$ with $512 \times 512$ data points. Underlying sectional profiles of the topographic images are taken at the dashed white line.

The capability of the force-feedback HSAFM was determined by observing how different imaging rates influence the imaging of the biological sample. Topographic images and their simultaneous force images taken under different imaging rates are displayed. Figures 5(a) and 5(b) show the images taken at $50 \mathrm{~ms} /$ line. The images were taken over a segment between two of the periodic indentations, which appears to be covered by a layer of biofilm. The biofilm is less compact than in Figure 4, with denser individual clusters evident by pockets of higher topographic elevation within the biofilm. The dense clusters in the thin film have a height $\sim 100$ $\mathrm{nm}$ and a diameter ranging $\sim 20-40 \mathrm{~nm}$ (as seen in the underlying sectional profiles). It is 
interesting to note that the denser portion of the film that is trapped in the lower left grating indentation with the oscillating-wave-like texture has a height of $\sim 200 \mathrm{~nm}$. This indicates that the denser the biofilm, the more the internal cells aggregate and the higher the topographic height is achieved. The lower right indentation is also covered by a thin film ( 40 $\mathrm{nm}$ height) so thinly layered that there are pockets where the height is merely $\sim 10 \mathrm{~nm}$ (in the dark strip with diameter of $\sim 800 \mathrm{~nm}$ ), although the film gets progressively larger near the upper terrace ( $200 \mathrm{~nm}$ height from the $\sim 40 \mathrm{~nm}$ ). In the top section of the upper terrace, small structures can be seen with heights of $\sim 20 \mathrm{~nm}$ and diameters of $\sim 10-50 \mathrm{~nm}$ (as seen previously in Figure 4, again these small features are presumably parts derived from the biofilm during transplantation). The highresolution topographic imaging is not degraded at all as the rate is increased from $50 \mathrm{~ms} /$ line to $20 \mathrm{~ms} /$ line. Most of the force images show that the force is constant on the upper terrace on the grating except when abrupt signal changes occur due to small features. However, when the imaging rate is accelerated, the force image becomes rougher due to the limiting capability of the piezo tube's response to the rapid signal change. In contrast to the topographic image, the force image (Figure 5(b)) again shows better contrast, which can be used as complementary information in understanding the delicate structural change on the biological surfaces. This higher contrast is again due to the residual force signals that were not compensated by the topographic feedback loop when abrupt changes occurred in sample's mechanical property or topography. The force images are analogous to frictional images in friction force microscopy (Baselt and Baldeschwieler, 1994; Overney et al., 1994) where sample's boundaries were always emphasized. For example, those delicate features in the force image cannot be easily identified with topographic image only. Figures 5(c) and 5(d) show the topographic and force images, respectively, taken at the same location with a different scan rate of $20 \mathrm{~ms} / \mathrm{line}$. No notable 
changes were discernible, except the appearance of a small y-axis striation pattern on the right side in the force image in Figure 5(d). The striation pattern has been observed previously and found to be due to the piezo tube approaching resonant vibration frequency (e.g. Ando et al., 2001; Ando et al., 2008a; Miyagi et al., 2008; Schitter et al., 2001; Sparks and Manalis, 2004). The pattern becomes more dominant as the scan rate increases. Figure 5(e) and Figure 5(f)) are the topographic and force images, respectively, taken with the scan rate of $10 \mathrm{~ms} / \mathrm{line}$. Both images show a larger striation pattern parallel to the y-axis. The images taken at the scan rate of $5 \mathrm{~ms} /$ line, (Figure 5(g) and Figure 5(h)) exhibit a greater increase in the striation pattern, particularly more prominent in the force image. Additionally, the sectional profile of Figure 5(g) shows decreased capability to image minute topographic variations. At $1 \mathrm{~ms} / \mathrm{line}$ (image not shown), features become too distorted to be accurately distinguishable due to the interference of the pattern in resolving the features on the images. The result suggests that the scanner resonance frequency is an important limiting factor deciding the speed limit of the force-feedback HSAFM. 

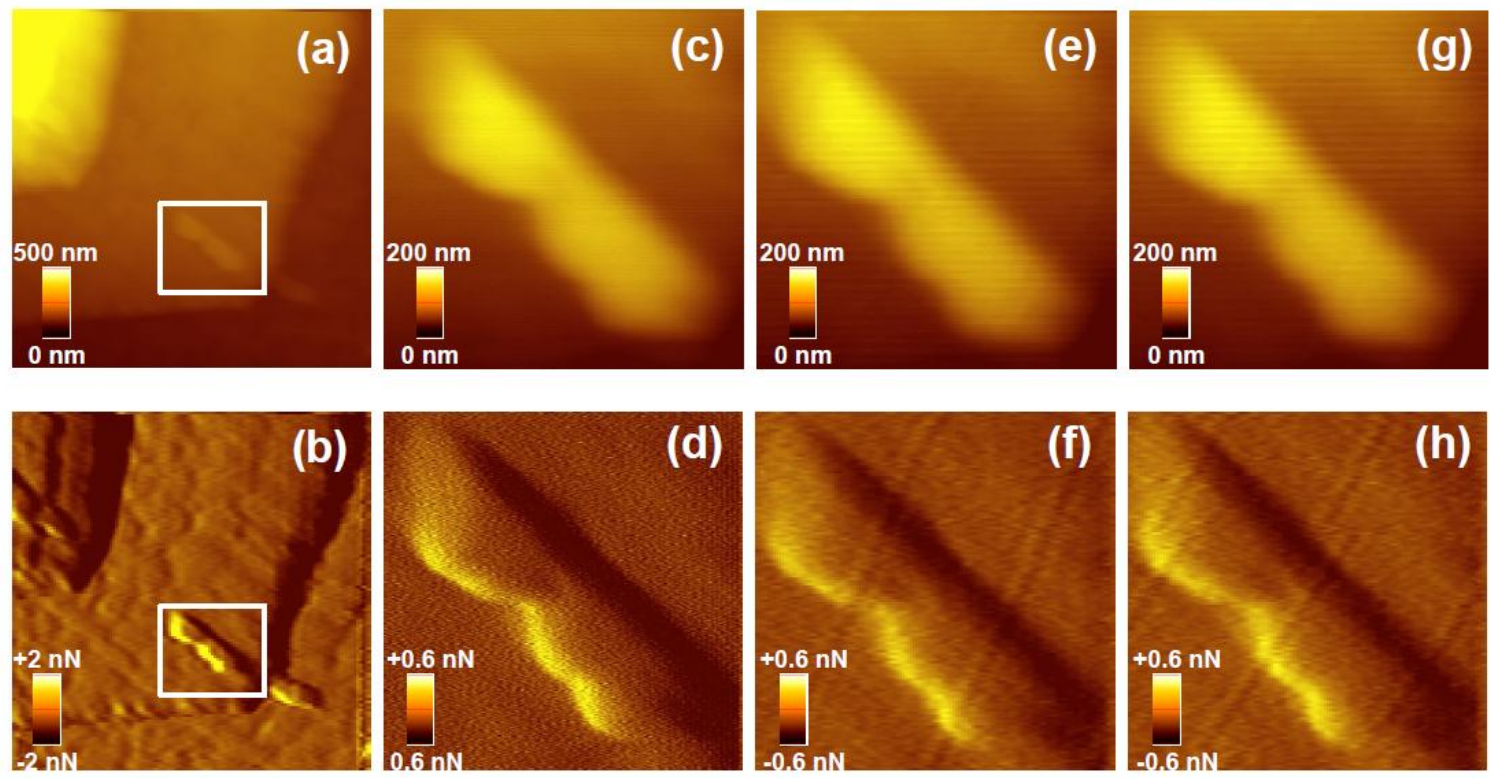

Figure 6. Topographical image (a) and force image (b) collected simultaneously at 0 $\mathrm{nN}$ set-force on the E. coli biofilms collected in the scan area of $8 \mu \mathrm{m} \times 8 \mu \mathrm{m}$ with 128 $\mathrm{x} 128$ data points. The scanning rate was selected to be $10 \mathrm{~ms} / \mathrm{line}$. Topographical and force images collected simultaneously at $0 \mathrm{nN}$ set-force in a reduced scan area of 2.5 $\mu \mathrm{m} \times 2.5 \mu \mathrm{m}$ to have a magnified image of an E. coli bacte/rium. Topographic and force images taken at $512 \times 512$ data points at a scan rate of $25 \mathrm{~ms} / \mathrm{line}(\mathbf{c}, \mathbf{d})$, at $128 \mathrm{x}$ 128 data points at a scanning rate of $10 \mathrm{~ms} / \mathrm{line}(\mathbf{e}, \mathbf{f})$, and at $128 \times 128$ data points at a scanning rate of $5 \mathrm{~ms} /$ line $(\mathbf{g}, \mathbf{h})$.

The invasiveness of the system during high-speed scanning was then tested on biological samples by imaging of what appears to be a single cell of $E$. coli on the grating sample (highlighted by the squared section) at different imaging rates. Figure 6(a) shows an $8 \mu \mathrm{m} \times 8$ $\mu \mathrm{m}$ high resolution topographic image and Figure 6(b) shows the corresponding force image, taken at $10 \mathrm{~ms} / \mathrm{line}$, of what appears to be a sequence of step-like biofilm structures (with each step height of $\sim 200 \mathrm{~nm}$ ) developed during the sample preparation. The image contains a small rod-shape about 2-3 $\mu \mathrm{m}$ long and about $0.5 \mu \mathrm{m}$ in diameter in the lower right corner. We considered the structure as a lone E. coli cell because the dimension and morphology is consistent with the literature values of E. coli (Alberts et al., 2002). We zoomed in on a squared area $(2.5 \mu \mathrm{m} \times 2.5 \mu \mathrm{m})$ outlined by white lines. Figures $\mathbf{6}(\mathbf{c})$ and $\mathbf{6}(\mathbf{d})$ shows topographic and 
force images collected simultaneously on the bacterium at an imaging rate of $25 \mathrm{~ms} / \mathrm{line}$. As expected with this scan rate, the images do not show any visible striation pattern. The E. coli was shown to have the characteristic smooth texture for the cell surface with a small indentation in the center of the cell. This difference in smoothness from those seen in the images of biofilm appears to be due to cell morphology being physiologically different when a cell is grown in a biofilm compared to a planktonic cell (freely floating cell) of the same organism (Allison, 2000; Lynch et al., 2003). When we increased the speeds to $10 \mathrm{~ms} / \mathrm{line}$ (see Figures $\mathbf{6}(\mathbf{e})$ and $\mathbf{6 ( f )})$, force images still did not show the striation pattern along the vertical y-direction. Interestingly, the striation pattern was still not visible when we increased the scan rate to $5 \mathrm{~ms} / \mathrm{line}$ (see Figures $\mathbf{6}(\mathrm{g})$ and $\mathbf{6}(\mathbf{h}))$. It appeared that the striation pattern is also dependent on the scan size as well as the scan rate, which is most likely due to the redirection of higher momentum when imaging a larger scan size at the same imaging rate (one frame per second). Since with larger scan sizes more distance must be covered in the same amount of time, the velocity while scanning must be greater. Therefore, the impulse or momentum change is greater when imaging at larger scanning sizes. The images of the E. coli were reproducible without showing any observable change of the $E$. coli structure even with the scan rate changes by a factor of five. This reproducibility indicated the absence of major deformation of the sample during the successive scans. This may be related to the fast response of force feedback and negligible small deformations within the sample's elastic regime between steps 3 and 4 in Figure 1. The interaction between a probing tip and a delicate surface (e.g., between a tip and gram-negative bacteria, which have a thin peptidoglycan layer compared to that of gram-positive bacteria) is known to even sometimes be irreproducible with conventional AFM imaging (Dufrêne, 2004). 


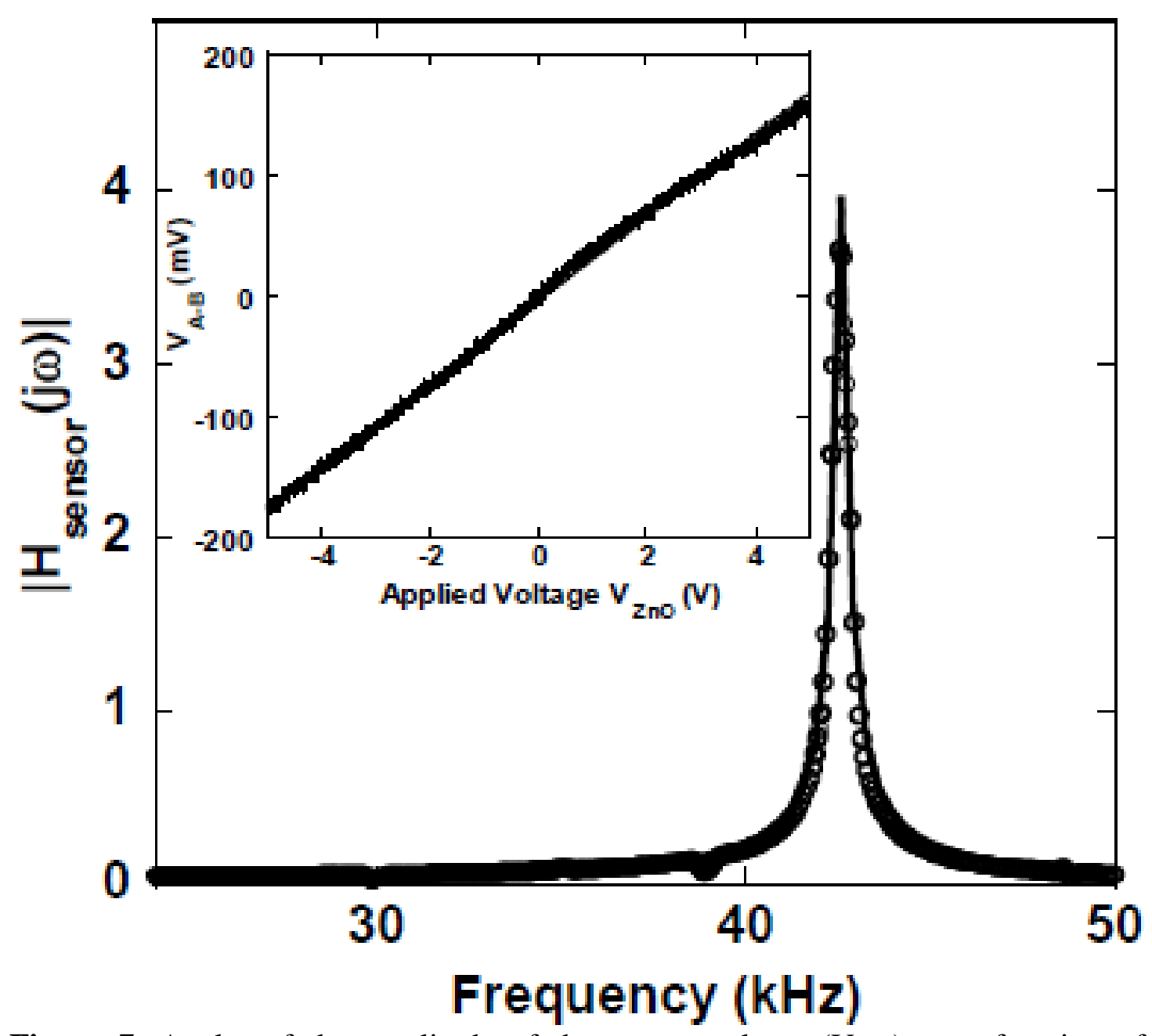

Figure 7. A plot of the amplitude of the output voltage $\left(\mathrm{V}_{\mathrm{A}-\mathrm{B}}\right)$ as a function of frequency $(f)$ of the sinusoidal driving voltage applied to the $\mathrm{ZnO}$ stack (solid line). The data was fitted with a classic second-order mechanical response to find the resonance frequency and damping constant. (inset) The deflection signal $\mathrm{V}_{\mathrm{A}-\mathrm{B}}$ as a function of the applied $\mathrm{ZnO}$ voltage signal $\left(\mathrm{V}_{\mathrm{ZnO}}\right)$ for the range between $-5 \mathrm{~V}$ and +5 $\mathrm{V}$ under the inactivation of force-feedback loop. The curve provides the dc gain constant $G_{Z n O}$.

The cantilever resonance frequency was determined as the crucial factor for the time scale of the DMASP cantilever in comparison with that of the piezo tube. Figure 7 shows a plot of the amplitude of the response signal as a function of the frequency measured with a lock-in amplifier. A driving signal with an $50 \mathrm{mV}$ amplitude was applied to the set-point input $\left(\mathrm{V}_{\text {Set-point }}\right.$ in Figure 2) for its frequency range between $1 \mathrm{~Hz}$ to $23 \mathrm{kHz}$ (TEMA: $2 \mathrm{MHz}$ function 
generator). One of the ADC inputs of the RHK 100 controller was used to record the measured amplitudes (Kim, 2009). The measured mechanical response data was fitted with a classic second-order mechanical response, $\left(G_{Z n O} \cdot \omega_{0}^{2}\right) / \sqrt{\left(\omega^{2}-\omega_{0}^{2}\right)^{2}+4\left(\zeta \omega_{0} \omega\right)^{2}}$ where $G_{Z n O}$ is dc gain constant, $\zeta$ is the damping ratio and $\omega_{0}$ is the natural resonance frequency. The solid line in Figure 7 is the curve fitted with the parameters with $\zeta$ of $3.5 \times 10^{-3}$ and $\omega_{0}$ of $2 \pi \times 42.6 \mathrm{kHz}$. The $G_{Z n O}$ was found to be $34.07 \mathrm{mV} / \mathrm{V}$ from the slope between the deflection signal $\left(\mathrm{V}_{\mathrm{A}-\mathrm{B}}\right)$ and the $\mathrm{ZnO}$ voltage signal $\left(\mathrm{V}_{\mathrm{ZnO}}\right)$, which was measured in advance (solid line in inset of Figure 7).

The cantilever resonance frequency of $42.6 \mathrm{kHz}$ mainly determined the bandwidth, as the bandwidth of the force-feedback controller is higher than $200 \mathrm{kHz}$. The shortest response time of the force-feedback is the inverse of the resonance frequency or $1 / f_{0}=23.4 \mu$. For the piezo tube, the resonance frequency was calculated from the observed striation pattern in Figure 5(g). Because the number of patterns is $\sim 15$ and the tip speed $5 \mathrm{~ms} /$ line, the periodicity is determined to be $0.33 \mathrm{~ms}$. This result corresponds to a $3 \mathrm{kHz}$ resonance frequency in the $\mathrm{x}-\mathrm{y}$ direction for this scanner. According to Zareian Jahromi et al., the axial resonant frequency tends to be approximately 3 to 3.5 times that of the bending resonant frequency (Zareian Jahromi et al., 2008). The axial resonance frequency is determined by $\sim 10 \mathrm{kHz}$ for this scanner. The shortest response time of the piezo tube is $\sim 1 / f_{0}=100 \mu$ s. So for example, Figure 6(a) has 128 data points per each line, the shortest time to complete a line scan is $12,800 \mu$ s. The fastest scan rate possible is $12.8 \mathrm{~ms} / \mathrm{line}$, which is roughly consistent with our observed scan rate of $10 \mathrm{~ms}$ per line. This consistency confirms that the low-resonance frequency of the piezo tube is the limiting factor that determines the bandwidth of the current force-feedback HSAFM. The imaging speed 
of the force-feedback HSAFM will be improved by using the piezo scanner with high-resonance frequencies such as conical piezo tubes (Rost et al., 2009).

Even with the use of a larger cantilever over the smaller-scale cantilever, the force-feedback HSAFM achieved high-speed imaging through the fast force-feedback mechanism. The spring constant is considered relatively infinite when combined with the force-feedback scheme. Due to the cooperativeness between the force-feedback loop and the piezo tube feedback loop, the system is able to capture distinguishable biological features at roughly one frame per second for an image with scan area $8 \mu \mathrm{m} \times 8 \mu \mathrm{m}$. The system presented in this paper uses the force between the cantilever probe and the sample as the input of the feedback for its imaging mechanism. In comparison, the tapping mode and force-modulation techniques use the amplitude of the cantilever motion as the input of their respective feedbacks in response to its interaction with the sample while applying a sinusoidal driving force. The force-feedback, to maintain the cantilever deflection constant, allows for faster response to topographic variations.

\section{Conclusion}

We designed and developed a HSAFM using force-feedback for imaging large-scale biological samples. The use of a normal-size, self-actuated cantilever avoids the arduous fabrication and signal detection with a smaller laser spot size associated with the use of a smaller cantilever. Three different images (a deflection image, a topographic image, and a force image) were collected simultaneously to have complementary information in biological studies. We demonstrated that the force-feedback HSAFM is capable of imaging $E$. coli and related biofilms at an imaging rate of one frame per second. The imaging rate was found to be limited by the resonance frequency of the piezo tube. Our further measurement of resonance frequency of the cantilever suggested that such imaging rates originated from the stiffness enhancement of the 
cantilever through the use of force-feedback. The HSAFM system using force-feedback is a novel approach for the high-speed imaging of large biological samples.

Acknowledgements: The authors gratefully acknowledge support from Dr. Kenneth A. Cornell for providing E. coli samples used for this study. This research was supported by NSF DMR1126854, NSF DBI-0852886, Faculty Research Initiation Grants (FRIG) and Collaborative Grant Improvement Initiative (CGII) at Boise State University. 


\section{REFERENCES}

Alberts, B., Johnson, A., Lewis, J., Raff, M., Roberts, K., Walter, P., 2002. Molecular Biology of The Cell, fourth ed. Garland Science, New York.

Albrecht, T. R., Grutter, P., Horne, D., Rugar, D., 1991. Frequency modulation detection using high-Q cantilevers for enhanced force microscope sensitivity. J. Appl. Phys. 69, 668-673.

Allison, D.G., 2000. Community structure and co-operation in biofilms. Cambridge University Press, Cambridge, UK.

Ando, T., Kodera, T., Takai, E., Maruyama, D., Saito, K., Toda, A., 2001. A high-speed atomic force microscope for studying biological macromolecules. Proc. Natl. Acad. USA 98, 12468-12472.

Ando, T., Uchihashi, T., Fukuma, T., 2008a. High-speed atomic force microscopy for nanovisualization of dynamic biomolecular process. Prog. Surf. Sci 83, 337-437.

Ando, T., Uchihashi, T., Kodera, N., Yamamoto, D., Taniguchi, M., Miyagi, A., Yamashita, H., 2008b. High-speed AFM and nano-visualization of biomolecular processes. Pflügers Archiv - Eur. J. Physiol. 456, 211-225.

Baselt, D.R., Baldeschwieler, J.D., 1994. Imaging spectroscopy with the atomic force microscope. J. Appl. Phys. 76, 33-38.

Bonander, J. R., Kim, B. I., 2008. Cantilever based optical interfacial force microscope. Appl. Phys. Lett. 92, 103124.

Crampton, N., Yokokawa, M., Dryden, D. T. F., Edwardson, J. M., Rao, D. N., Takeyasu, K., Yoshimura, S. H., Henderson, R. M., 2007. Fast-scan atomic force microscopy reveals that the type III restriction enzyme EcoP15I is capable of DNA translocation and looping. Proc. Natl. Acad. USA 104, 12755 - 12760. 
Dufrêne, Y. F., 2004. Using nanotechniques to explore microbial surfaces. Nat. Rev. Microbiol. $2,451-460$.

Fantner, G.E., Barbero, R.J., Gray, D.S., Belcher, A.M., 2010. Kinetics of antimicrobial peptide activity measured on individual bacteria cells using high-speed atomic force microscopy. Nat. Nanotechnol. 5, 280-285.

Hansma, P.K., Schitter, G., Fantner, G.E., Prater, C., 2006. High-speed atomic force microscopy. Science. 314, 601-602.

Humphris, A. D. L, Miles, M. J., Hobbs, J. K., 2005. A mechanical microscope: high-speed atomic force microscopy. Appl. Phys. Lett. 86, 034106.

Katan, A. J., Dekker, C., 2011. High-speed AFM reveals the dynamics of single biomolecules at the nanometer scale. Cell. 147, 979-982.

Kim, B. I., 2009. Separation of topographic features from magnetic force images using capacitive coupling effect. Rev. Sci. Instrum. 80, 023702.

Kim, B. I., Bonander, J. R., Rasmussen, J. A., 2011a. Simultaneous measurement of normal and friction forces using a cantilever-based optical interfacial force microscope. Rev. Sci. Instrum. 82, 053711.

Kim, B. I., Rasmussen, J. A., Kim, E. J., 2011b. Large oscillatory forces generated by interfacial water under lateral modulation between two hydrophilic surfaces. Appl. Phys. Lett. 99, 201902.

Kodera, N., Sakashita, M., Ando, T., 2006. Dynamic proportional-integral-differential controller for high-speed atomic force microscopy. Rev. Sci. Instrum. 77, 083704.

Lynch, J.F., Lappin-Scott, Hilary, M., Costerton, J.W., 2003. Microbial biofilms. Cambridge University Press, Cambridge, UK. 
Manalis, S. R., Minne, S. C., Quate, C. F., 1996a. Atomic forc microscopy for high speed imaging using cantilevers with an integrated actuator and sensor. Appl. Phys. Lett. 68, 871-873.

Manalis, S. R., Minne, S. C., Atalar, A., Quate, C. F., 1996b. Highspeed atomic force microscopy using an integrated actuator and optical lever detection. Rev. Sci. Instrum. 67, 3294-3297.

Meyer, E., Heinzelmann, H., Grütter, P., Jung, T. H., Weisskopf, T., Hidber, H. R., Lapka, R., Rudin, H., Güntherodt, H. J., 1988. Comparative study of lithium fluoride and graphite by atomic force microscopy (AFM). J. Microsc.-Oxford 152, 269-280.

Minne, S. C., Manalis, S. R., Quate, C. F., 1995. Parallel atomic force microscopy using cantilevers with integrated piezoresistive sensors and integrated piezoelectric actuators. Appl. Phys. Lett, 67, 3918-3920.

Miyagi, A., Tsunaka, Y., Uchihashi, T., Mayanagi, K., Hirose, S., Morikawa, K., Ando, T., 2008. Visualization of intrinsically disordered regions of proteins by high-speed atomic force microscopy. Chem. Phys. Chem. 9, 1859-1866

Overney, R.M., Bonner, T., Meyer,E., Ruöetschi, M., Luöthi, R., Howald, L., Frommer, J., Guöntherodt, H.-J., Fujihira, M., Takano, H., 1994. Elasticity, wear, and friction properties of thin organic films observed with atomic force microscopy. J. Vac. Sci. Technol. 12, 1973-1976.

Picco, L. M., Bozec, L., Ulcinas, A., Engledew, D. J., Antognozzi, M., Horton, M. A., Miles, M. J., 2007. Breaking the speed limit with atomic force microscopy. Nanotechnology 18 , 044030-044033. 
Rogers, B., York, D., Whisman, N., Jones, M., Murray, K., Adams, J.D., Sulchek, T., Minne, S.C., 2002. Tapping mode atomic force microscopy in liquid with an insulated piezoelectric micro-actuator. Rev. Sci. Instrum. 73, 3242-3244.

Rogers, B., Sulchek, T., Murray, K., York, D., Jones, M., Manning, L., Malekos, S., Beneschott, B., Adams, J. D., Cavazos, H., Minne, S. C., 2003. High speed tapping mode atomic force microscopy in liquid using an insulated piezoelectric cantilever. Rev Sci Instrum $74,4683-4686$.

Rost, M. J., van Baarle, G. J. C., Katan, A. J., van Spengen, W. M., Schakel, P., van Loo, W. A., Oosterkamp, T. H., Frenken, J. W. M., 2009. Video-rate scanning probe control challenges: setting the stage for a microscopy revolution. Asian J. Control 11, 110-129.

Schitter, G., Menold, P., Knapp, H., Allgöwer, F., Stemmer, A., 2001. High performance feedback for fast scanning atomic force microscopes. Rev. Sci. Instrum. 72, 3320-3931.

Sparks A. W., Manalis, S. R., 2004. Scanning probe microscopy with inherent disturbance suppression. Appl. Phys. Lett. 85, 3929-3931.

Sulchek, T., Minne, S.C., Adams, J.D., Fletcher, D.A., Atalar, A., Quate, C.F., Adderon, D.M., 1999. Dual integrated actuators for extended range high speed atomic force microscopy. Appl. Phys. Lett. 75, 1637-1639.

Sulchek, T., Hsieh, R., Adams, J. D., Yaralioglu, G. G., Minne, S. C., Quate, C. F., Cleveland, J. P., Atalar, A., Adderton, D. M., 2000. High-speed tapping mode imaging with active Q control for atomic force microscopy. Appl. Phys. Lett. 76, 1473-1475.

van Noort, S.J, van Der Werf, K. O., de Grooth, B. G., Greve, J., 1999. High speed atomic force microscopy of biomolecules by image tracking. Biophys J. 77, 2295-2303.

Veeco, Probes and Accessories (Santa Barbara, CA 2006), pp. 88. 
Viani, M.B., Schaffer, T.E., Paloczi, G.T., Pietrasanta, I., Smith, B.L., Thompson, J.B., Richter, M., Rief, M., Gaub, H.E., Plaxco, K.W., Cleland, A.N., Hansma, H.G., Hansma, P.K., 1999. Fast imaging and fast force spectroscopy of single biopolymers with a new atomic force microscope designed for small cantilevers. Rev. Sci. Instrum. 70, 4300-4303.

Zareian Jahromi, S. A., Salomons, M., Sun, Q., Wolkow, R. A., 2008. Prediction of the resonant frequency of piezoelectric tube scanners through three-dimensional finite element modeling of a tube assembly. Rev. Sci. Instrum. 79, 076104.

Zou, Q., Leang, K.K., Sadoun, E., Reed, M.J., Devasia, S., 2008. Control issues in high-speed AFM for biological applications: collagen imaging example. Asian J. Control. 6, 164178. 